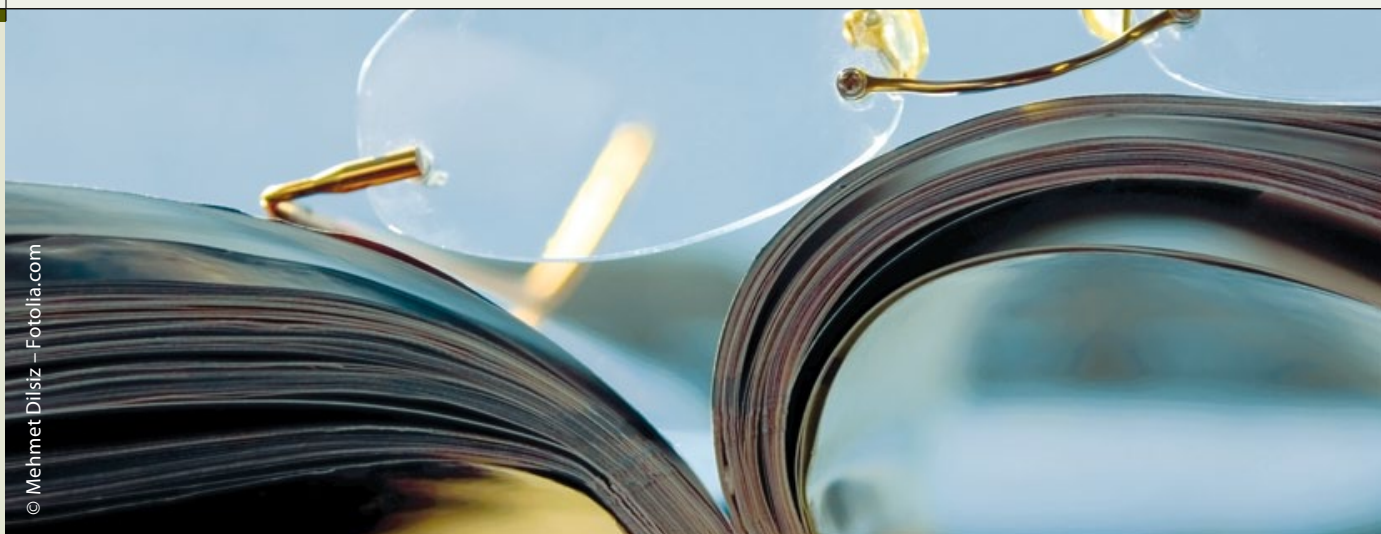

\title{
Vorsicht vor Missbrauch: Analgetika gegen die Trübsal
}

\section{Depressionen und chronische Schmerzen - diese Konstellation kann für Patienten riskant werden. Denn dann greifen sie offenbar öfter zu Opioiden als ratsam wäre.}

atienten mit chronischen Schmerzen, die zugleich Depressionen haben, sind besonders anfällig für den Missbrauch von Opioiden. Sie nehmen die Schmerztabletten häufig auch gegen Schlafstörungen oder gegen Stress und tendieren außerdem zu höheren Dosierungen als der Arzt ihnen verordnet hat. Das berichtet ein kanadisches Forscherteam, das eine Telefonumfrage organisiert hatte.

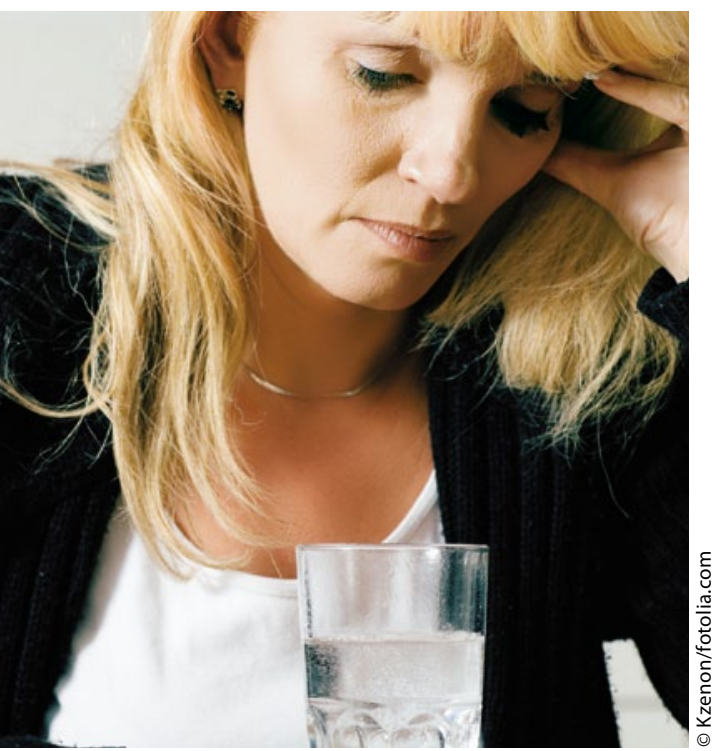

Bei Schmerzen geht es oft nicht ohne Opioide. Patienten mit zusätzlicher Depression neigen häufiger zur Überdosierung dieser Schmerzmittel.
Befragt wurden insgesamt 1.300 Patienten mit chronischen Schmerzen, bei denen bis dato kein Substanzmissbrauch bekannt war. Von diesen waren $30 \%$ leicht, $20 \%$ mittelschwer und $18 \%$ schwer depressiv gemäß dem PHQScore. Das Akronym steht für Patient Health Questionnaire. Bei den Schmerzpatienten, die gleichzeitig unter schweren Depressionen litten, war die Wahrscheinlichkeit, dass sie die vom Arzt verschriebenen Opioide auch gegen Symptome wie Schlafstörungen oder Stress einsetzten, um mehr als das Doppelte erhöht ( $\mathrm{p}=0,001)$. Bei Patienten mit mittelschweren Depressionen lag dieses Risiko 1,75-mal höher als bei nicht depressiven Teilnehmern $(\mathrm{p}=0,031)$.

Auch die Einnahme höherer Opioiddosen als verordnet kam bei den depressiven Schmerzpatienten besonders häufig vor. Dieses Risiko stieg schon bei leichten Anzeichen einer Depression um fast das Doppelte (Faktor 1,93; $\mathrm{p}<0,001$ ). Bei mittelschwerer Depression war die Wahrscheinlichkeit nahezu dreifach und bei schweren Depressionen um mehr als das 3-Fache erhöht (Faktor 2,89 bzw. 3,13; p jeweils $<0,001$ ).

Insgesamt zeigte sich mit zunehmender Schwere der Depressionen ein verstärkter Trend zum Missbrauch. So stieg der Anteil der Patienten, die Opioide einnahmen, um damit andere Beschwerden als den Schmerz zu lindern, von
$40 \%$ bei den leicht Depressiven auf $47 \%$ bei den mittelschwer und $52 \%$ bei den schwer Depressiven (zum Vergleich: $37 \%$ in der Gruppe ohne Depressionen). Mit dem Grad der Depression verknüpft war auch der Trend zum Überdosieren: $43 \%$ der Patienten mit leichten Depressionen nahmen zu große Mengen des Opioids ein, bei den mittelschwer und schwer Depressiven waren es 54 bzw. $58 \%$. Unter den nicht depressiven Schmerzpatienten hielten sich $27 \%$ nicht an die empfohlene Dosis.

Fazit: Dass Opioide neben schmerzlindernden auch anxiolytische Eigenschaften haben und zudem die Stimmung heben können, ist bekannt. Dies wäre eine Erklärung dafür, dass gerade Patienten mit Depressionen zum Missbrauch neigen, vermuten Studienautorin Alicia Grattan und Kollegen von der Universität des Bundesstaates Washington in Seattle.

Möglicherweise verhält es sich aber auch entgegengesetzt, und die Opioide selbst befördern die Depression, indem sie den Patienten lethargisch machen. Wie dem auch sei, bei der Behandlung depressiver Schmerzpatienten mit Opioiden müsse man das erhöhte Missbrauchspotenzial berücksichtigen und auch die Depression in jedem Fall ausreichend therapieren, schreiben die Forscher.

Grattan A et al. Depression and prescription opioid misuse among chronic opioid therapy recipients with no history of substance abuse. Ann Fam Med. 2012;10(4):304-11. 